\title{
A Class of Coupled Causal Differential Equations
}

\author{
Wen-Li Wang ${ }^{1}$, Jing-Feng Tian ${ }^{2, * \mathbb{D}}$ and Wing-Sum Cheung ${ }^{3}$ \\ 1 Faculty of Fundamental Courses, China University of Geosciences Great Wall College, Baoding 071000, \\ China; emilyzh@163.com \\ 2 College of Science and Technology, North China Electric Power University, Baoding 071051, China \\ 3 Department of Mathematics, University of Hong Kong, Pokfulam Road, Hong Kong, China; \\ wscheung@hku.hk \\ * Correspondence: tianjf@ncepu.edu.cn
}

Received: 20 August 2018; Accepted: 15 September 2018; Published: 20 September 2018

check for updates

\begin{abstract}
In this thesis, we consider the existence of extreme solutions to a class of coupled causal differential equations. By utilizing two comparison theorems and a monotone iterative technique, we have obtained sufficient conditions under which the equations have extreme solutions. One practical example is presented.
\end{abstract}

Keywords: existence results; causal operators; causal differential equations; coupled lower and upper approximative solutions; monotone iterative technique

\section{Introduction}

We first study the following causal differential equations:

$$
\left\{\begin{array}{l}
x^{\prime}(t)=Q_{1}(x, y)(t), \quad t \in I, \\
y^{\prime}(t)=Q_{2}(y, x)(t), \quad t \in I, \\
g_{1}(x(0), x(Y), y(0), y(Y))=0, \\
g_{2}(y(0), y(Y), x(0), x(Y))=0,
\end{array}\right.
$$

where $I=[0, Y], E=C\left(I^{2}, \mathbb{R}\right), Q_{1}, Q_{2} \in C(E, E)$ are causal operators and $g_{1}, g_{2} \in C\left(\mathbb{R}^{4}, \mathbb{R}\right)$ and $Q_{1}, Q_{2}$ are bounded. The term causal operator first appeared in Volterra's work on the integral equations. It is a non-anticipative operator, and it was first applied by Volterra. The definition of the operator was given by Tonelli and Tychonoff. This theory can unify many types of equations. Peer works were reported by Drici et al. [1] and Geng [2]. Compared with traditional models, causal differential equations are more similar to real-world problems and more widely used. It has been tapped in depth by many authors in these years. Interested readers are referred to the monograph [3] and article [4] for more details.

At the same time, the research of a class of coupled differential equations is also crucial as such a system often occurs in real-life applications; it is a physical model of phenomena, in terms of the fluid dynamic traffic model, oscillations of earthquakes and economics and has attracted much attention; see, e.g., [5-7]. It should be noted that, in [8], Jankowski discussed coupled causal difference equations. The author developed the monotone iterative technique and gained the existence of extreme solutions to such equations. Affected by the above results, we study a class of coupled causal differential equations and obtain the extreme solutions.

The following is the frame of this paper. In Section 2, two comparison theorems are presented. In Section 3, we investigate the linear differential equations of (1). In Section 4, we establish the existence of the extremal solutions to (1). In the end, a computed example is given to verify the outcome. 


\section{Comparison Results}

In this part, we set $E=C(I, \mathbb{R})$ and we define:

$$
\|f-g\|:=\max _{t \in I}|f(t)-g(t)| .
$$

Let $\Omega:=E \cap C^{1}(I, \mathbb{R})$. A pair $(x, y), x, y \in \Omega$ is treated as a solution to Problem (1) if it contains (1).

Definition 1. Suppose that $Q \in C(E, E)$ is called a causal operator if for every pair of elements in $E$ such that $x_{i}(s)=y_{i}(s), i=1,2$ for $t_{0} \leq s \leq t \leq Y$, then:

$$
Q\left(x_{1}, x_{2}\right)(s)=Q\left(y_{1}, y_{2}\right)(s), \quad t_{0} \leq s \leq t .
$$

Lemma 1. Suppose that $z \in \Omega$ satisfies:

$$
\left\{\begin{array}{l}
z^{\prime}(t) \leq-A(t) z(t)-(\mathcal{L} z)(t), \quad t \in I, \\
z(0) \leq \lambda z(Y),
\end{array}\right.
$$

where $A \in C(I,[0,+\infty))$ and $\mathcal{L} \in C(E, E)$ is a positive linear operator satisfying:

$$
\int_{0}^{Y}[A(t)+\mathcal{L}(t)] d t \leq \epsilon, \quad 0<\epsilon \leq 1 .
$$

then $z(t) \leq 0, t \in I$.

Proof. Let us say $z(t) \leq 0 \forall t \in I$ is not true; one has two situations:

Case 1. There exists $\bar{t} \in I$ that meets $z(\bar{t})>0, z(t) \geq 0, t \in I$.

Then, from (2), we have $z^{\prime}(t) \leq 0$ for $t \in I$; hence, $z(t)$ is nonincreasing on $I$. If $\epsilon=1, z(0) \leq z(Y)$ shows that $z(t) \equiv$ is constant, so $z^{\prime}(t) \equiv 0$. As $z(\bar{t})>0$, one has $0 \equiv z^{\prime}(\bar{t}) \leq-A(t) z(\bar{t})-(\mathcal{L} z)(\bar{t})<0$, which is a conflict. If $0<\epsilon<1$, then $z(Y) \leq z(0) \leq \epsilon z(Y)$, so it is also a conflict.

Case 2. There are $t_{m}$ and $t_{n} \in I$ satisfying $z\left(t_{m}\right)<0$ and $z\left(t_{n}\right)>0$.

Put $\inf _{t \in I} z(t)=-r, r>0$. With generality, we may assume that $z\left(t_{m}\right)=-r$.

Subcase (a). If $t_{m}<t_{n}$, from (2), we gain:

$$
\begin{aligned}
z\left(t_{n}\right)-z\left(t_{m}\right) & =\int_{t_{m}}^{t_{n}} z^{\prime}(t) d t \leq-\int_{t_{m}}^{t_{n}}[A(t) z(t)+(\mathcal{L} z)(t)] d t \\
& \leq r\left\{\int_{0}^{Y}[A(t)+\mathcal{L}(t)] d t\right\}
\end{aligned}
$$

hence:

$$
\left.0<z\left(t_{n}\right) \leq-r+r \int_{0}^{Y}[A(t)+\mathcal{L}(t)] d t\right]=r\left[\int_{0}^{Y}[A(t)+\mathcal{L}(t)] d t-1\right]
$$

which yields:

$$
\int_{0}^{Y}[A(t)+\mathcal{L}(t)] d t>1
$$

which contradicts (3).

Subcase (b). If $t_{m}>t_{n}$, from (2), we get:

$$
z(Y)=z\left(t_{m}\right)+\int_{t_{m}}^{Y} z^{\prime}(t) d t \leq z\left(t_{m}\right)+r \int_{t_{m}}^{Y}[A(t)+\mathcal{L}(t)] d t
$$

and:

$$
z\left(t_{n}\right)=z(0)+\int_{0}^{t_{n}} z^{\prime}(t) d t \leq z(0)+r \int_{0}^{t_{n}}[A(t)+\mathcal{L}(t)] d t .
$$


Using the fact $z(0) \leq \epsilon z(Y), 0<\epsilon \leq 1$, we obtain:

$$
\begin{aligned}
0<z\left(t_{n}\right) & \leq \epsilon\left(z\left(t_{m}\right)+r \int_{t_{m}}^{Y}[A(t)+\mathcal{L}(t)] d t\right)+r \int_{0}^{t_{n}}[A(t)+\mathcal{L}(t)] d t \\
& \leq-r \epsilon+r\left\{\int_{0}^{Y}[A(t)+\mathcal{L}(t)] d t\right\} \\
& =r\left\{\int_{0}^{Y}[A(t)+\mathcal{L}(t)] d t-\epsilon\right\},
\end{aligned}
$$

which is a conflict with a given condition. Therefore, $z(t) \leq 0, t \in J$. The proving process is done.

Lemma 2. Let $j, k \in \Omega$ be such that:

$$
\left\{\begin{aligned}
& j^{\prime}(t) \leq-A_{1}(t) j(t)+A_{2}(t) k(t)-\left(\mathcal{L}_{1} j\right)(t)+\left(\mathcal{L}_{2} k\right)(t), \quad t \in I, \\
& k^{\prime}(t) \leq-A_{1}(t) k(t)+A_{2}(t) j(t)-\left(\mathcal{L}_{1} k\right)(t)+\left(\mathcal{L}_{2} j\right)(t), \quad t \in I, \\
& j(0) \leq \epsilon_{1} j(Y)+\epsilon_{2} k(Y) \\
& k(0) \leq \epsilon_{1} k(Y)+\epsilon_{2} j(Y)
\end{aligned}\right.
$$

where $0<\epsilon_{1}+\epsilon_{2} \leq 1,0<\epsilon_{1}-\epsilon_{2} \leq 1, A_{1}, A_{2} \in C(I,[0,+\infty)), A_{1}(t) \geq A_{2}(t), t \in I, \mathcal{L}_{1}-\mathcal{L}_{2} \in C(E, E)$ and $\mathcal{L}_{1}+\mathcal{L}_{2} \in C(E, E)$ are positive linear operators. Additionally,

$$
\begin{aligned}
& \max \left\{\frac{\int_{0}^{Y}\left[A_{1}(t)+A_{2}(t)+\mathcal{L}_{1}(t)+\mathcal{L}_{2}(t)\right] d t}{\epsilon_{1}-\epsilon_{2}}, \frac{\int_{0}^{Y}\left[A_{1}(t)-A_{2}(t)+\mathcal{L}_{1}(t)-\mathcal{L}_{2}(t)\right] d t}{\epsilon_{1}+\epsilon_{2}}\right\} \\
& \leq 1
\end{aligned}
$$

Then, $j(t) \leq 0$ and $k(t) \leq 0, t \in I$.

Proof. Let $c(t)=j(t)+k(t), t \in I$. Then:

$$
\begin{aligned}
c^{\prime}(t)=j^{\prime}(t)+k^{\prime}(t) & \leq-A_{1}(t) c(t)+A_{2}(t) c(t)-\left[\left(\mathcal{L}_{1}-\mathcal{L}_{2}\right) c\right](t) \\
& \leq-\left[A_{1}(t)-A_{2}(t)\right] c(t)-\left[\left(\mathcal{L}_{1}-\mathcal{L}_{2}\right) c\right](t), \quad t \in I
\end{aligned}
$$

and:

$$
c(0)=j(0)+k(0) \leq \epsilon_{1} c(Y)+\epsilon_{2} c(Y)=\left(\epsilon_{1}+\epsilon_{2}\right) c(Y) .
$$

From Lemma (1), $c(t) \leq 0$. Hence, $j(t) \leq-k(t)$ and $k(t) \leq-j(t)$, then we have:

$$
\left\{\begin{array}{l}
j^{\prime}(t) \leq-\left[A_{1}(t)+A_{2}(t)\right] j(t)-\left[\left(\mathcal{L}_{1}+\mathcal{L}_{2}\right) j\right](t), \quad t \in I, \\
j(0) \leq\left(\epsilon_{1}-\epsilon_{2}\right) j(Y)
\end{array}\right.
$$

and:

$$
\left\{\begin{array}{l}
k^{\prime}(t) \leq-\left[A_{1}(t)+A_{2}(t)\right] k(t)-\left[\left(\mathcal{L}_{1}+\mathcal{L}_{2}\right) k\right](t), \quad t \in I, \\
k(0) \leq\left(\epsilon_{1}-\epsilon_{2}\right) k(Y) .
\end{array}\right.
$$

From Lemma (1), one obtains $j(t) \leq 0, k(t) \leq 0, t \in I$. This is the end.

\section{Existence Results}

Let us consider the linear problem:

$$
\left\{\begin{array}{l}
x^{\prime}(t)=-A(t) x(t)-(\mathcal{L} x)(t)+\sigma(t), \quad t \in I \\
N_{1} x(0)-N_{2} x(Y)=B
\end{array}\right.
$$

where $N_{1}, N_{2}$ are constants, $\sigma \in E, A \in C(I,[0,+\infty)), B \in \mathbb{R}$. 
Lemma 3. $x \in \Omega$ is one solution of (6) when $x \in E$ is one solution of the equation:

$$
x(t)=\frac{B e^{-\int_{0}^{t} A(r) d r}}{N_{1}-N_{2} e^{-\int_{0}^{Y} A(r) d r}}+\int_{0}^{Y} D(t, s)[\sigma(s)-(\mathcal{L} x)(s)] d s,
$$

where $B=-g(\eta(0), \eta(T))+N_{1} \eta(0)-N_{2} \eta(Y), \eta \in \Omega, A \in C(I,[0,+\infty)), N_{1}, N_{2}$ are constants with $N_{1} \neq N_{2} e^{-\int_{0}^{Y} A(r) d r}$, and:

$$
D(t, s):= \begin{cases}\frac{N_{2}}{N_{1}-N_{2} e^{-\int_{0}^{Y} A(r) d r}} e^{\int_{t}^{0} A(r) d r} e^{\int_{Y}^{s} A(r) d r}+e^{\int_{t}^{s} A(r) d r}, & 0 \leq s<t \leq Y, \\ \frac{N_{2}}{N_{1}-N_{2} e^{-\int_{0}^{Y} A(r) d r}} e^{\int_{t}^{0} A(r) d r} e^{\int_{Y}^{s} A(r) d r}, & 0 \leq t \leq s \leq Y .\end{cases}
$$

Proof. Suppose that $x \in \Omega$ is one solution of Problem (6), one achieves:

$$
x(t)=x(0) e^{-\int_{0}^{t} A(r) d r}+\int_{0}^{t} e^{\int_{t}^{s} A(r) d r}[\sigma(s)-(\mathcal{L} x)(s)] d s
$$

Let $t=Y$; we get:

$$
x(Y)=x(0) e^{-\int_{0}^{Y} A(r) d r}+\int_{0}^{Y} e^{\int_{Y}^{s} A(r) d r}[\sigma(s)-(\mathcal{L} x)(s)] d s .
$$

Since $N_{1} x(0)-N_{2} x(T)=B$, we obtain:

$$
\begin{aligned}
x(0)= & \frac{B}{N_{1}-N_{2} e^{-\int_{0}^{Y} A(r) d r}} \\
& \quad+\frac{N_{2}}{N_{1}-N_{2} e^{-\int_{0}^{Y} A(r) d r}} \int_{0}^{Y} e^{\int_{Y}^{s} A(r) d r}[\sigma(s)-(\mathcal{L} x)(s)] d s .
\end{aligned}
$$

Then:

$$
x(t)=\frac{B e^{-\int_{0}^{t} A(r) d r}}{N_{1}-N_{2} e^{-\int_{0}^{Y} A(r) d r}}+\int_{0}^{Y} D(t, s)[\sigma(s)-(\mathcal{L} x)(s)] d s,
$$

where:

$$
D(t, s)= \begin{cases}\frac{N_{2}}{N_{1}-N_{2} e^{-\int_{0}^{Y} A(r) d r}} e^{\int_{t}^{0} A(r) d r} e^{\int_{Y}^{s} A(r) d r}+e^{\int_{t}^{s} A(r) d r}, & 0 \leq s<t \leq Y, \\ \frac{N_{2}}{N_{1}-N_{2} e^{-\int_{0}^{Y} A(r) d r}} e^{\int_{t}^{0} A(r) d r} e^{\int_{Y}^{s} A(r) d r}, & 0 \leq t \leq s \leq Y .\end{cases}
$$

Apparently, if $x(t)$ is a solution of (6), so $x(t)$ will also be a solution of (7). The proof is complete.

Clearly, $\|D(t, s)\|=\max \left\{\left|\frac{N_{1}}{N_{1}-N_{2} e^{-\int_{0}^{Y} A(r) d r}}\right|,\left|\frac{N_{2}}{N_{1}-N_{2} e^{-\int_{0}^{Y} A(r) d r}}\right|\right\}$. In the next part, we define $\|D(t, s)\|=d$.

Lemma 4. $A \in C(I,[0,+\infty)), N_{1} \neq N_{2} e^{-\int_{0}^{Y} A(r) d r}$, and:

$$
d\|\mathcal{L}\| Y<1
$$

Then, (6) has only one solution. 
Proof. Give a mapping $F: E \rightarrow E$ by:

$$
(F x)(t):=\frac{B e^{-\int_{0}^{t} A(r) d r}}{N_{1}-N_{2} e^{-\int_{0}^{Y} A(r) d r}}+\int_{0}^{Y} D(t, s)[\sigma(s)-(\mathcal{L} x)(s)] d s .
$$

For any $x_{1}, x_{2} \in E$, we have:

$$
\left\|F x_{1}-F x_{2}\right\| \leq d\|\mathcal{L}\| Y\left\|x_{2}-x_{1}\right\| .
$$

From the Banach contraction principle, (6) has only one solution. The proof is then finished.

Remark 1. If $A \in C(I,[0,+\infty)), N_{1} \geq N_{2}>0$, and:

$$
\frac{N_{1}}{N_{1}-N_{2} e^{-\int_{0}^{Y} A(r) d r}}\|\mathcal{L}\| Y<1 .
$$

Then, (6) has only one solution.

Let $B(k, h)(t):=A_{1}(t) k(t)+A_{2}(t) h(t)+\left(\mathcal{L}_{1} k\right)(t)+\left(\mathcal{L}_{2} h\right)(t)$.

Theorem 1. Suppose that linear operators $\mathcal{L}_{1}-\mathcal{L}_{2} \in C(E, E)$ and $\mathcal{L}_{1}+\mathcal{L}_{2} \in C(E, E)$ are positive. Let $\sigma_{1}, \sigma_{2} \in E, C_{1}, C_{2} \in \mathbb{R}, A_{1}, A_{2} \in C(I,[0,+\infty))$ and $A_{1}(t) \geq A_{2}(t)$ for $t \in I$. Moreover, we assume that (5) holds, $N_{1}, N_{2}, N_{3}$ are constants with $N_{1} \neq\left(N_{2}-N_{3}\right) e^{-\int_{0}^{Y}\left[A_{1}(r)+A_{2}(r)\right] d r}$, $N_{1} \neq\left(N_{2}+N_{3}\right) e^{-\int_{0}^{Y}\left[A_{1}(r)-A_{2}(r)\right] d r}$, and:

$$
\max \left\{\frac{N_{1}\left\|\mathcal{L}_{1}+\mathcal{L}_{2}\right\| Y}{N_{1}-\left(N_{2}-N_{3}\right) e^{-\int_{0}^{Y}\left[A_{1}(r)+A_{2}(r)\right] d r}}, \frac{N_{1}\left\|\mathcal{L}_{1}-\mathcal{L}_{2}\right\| Y}{N_{1}-\left(N_{2}+N_{3}\right) e^{-\int_{0}^{Y}\left[A_{1}(r)-A_{2}(r)\right] d r}}\right\}<1 .
$$

Then, the system:

$$
\left\{\begin{array}{l}
k^{\prime}(t)=-B(k, h)(t)+\sigma_{1}(t), \quad t \in I \\
h^{\prime}(t)=-B(h, k)(t)+\sigma_{2}(t), \quad t \in I \\
N_{1} x(0)=N_{2} k(Y)-N_{3} h(Y)+A_{1} \\
N_{1} y(0)=N_{2} h(Y)-N_{3} k(Y)+A_{2}
\end{array}\right.
$$

has only one solution.

Proof. Set $e=k+h, l=k-h$. Then, we have:

$$
\left\{\begin{array}{l}
e^{\prime}(t)=-\left[A_{1}(t)+A_{2}(t)\right] e(t)-\left[\left(\mathcal{L}_{1}+\mathcal{L}_{2}\right) e\right](t)+\sigma_{1}(t)+\sigma_{2}(t) \\
N_{1} e(0)=\left(N_{2}-N_{3}\right) e(Y)+C_{1}+C_{2}
\end{array}\right.
$$

and:

$$
\left\{\begin{array}{l}
l^{\prime}(t)=-\left[A_{1}(t)-A_{2}(t)\right] l(t)-\left[\left(\mathcal{L}_{1}-\mathcal{L}_{2}\right) l\right](t)+\sigma_{1}(t)-\sigma_{2}(t), \\
N_{1} l(0)=\left(N_{2}+N_{3}\right) l(Y)+C_{1}-C_{2} .
\end{array}\right.
$$

By Lemma 4, both (10) and (11) have a unique solution.

Note that $k=\frac{e+l}{2}, h=\frac{e-l}{2}$, and the pair $(k, h)$ is the only solution of Problem (9). This ends the proof. 


\section{Main Results}

Definition 2. A pair $(S, W)$ is seen as a coupled lower and upper approximative solution to (1) if:

$$
\left\{\begin{array}{l}
S^{\prime}(t) \leq Q_{1}(S, W)(t), \quad t \in I \\
W^{\prime}(t) \geq Q_{2}(W, S)(t), \quad t \in I \\
g_{1}(S(0), S(Y), W(0), W(Y)) \leq 0 \\
g_{2}(W(0), W(Y), S(0), S(Y)) \geq 0
\end{array}\right.
$$

Theorem 2. Suppose that (5), (8) hold, $Q_{1}, Q_{2} \in C(E, E)$ and

$\left(H_{1}\right)$ the function $(S, W) \in \Omega$ is the coupled lower and upper approximative solution to Problem (1) satisfying $S(t) \leq W(t), t \in I ;\left(H_{2}\right)$ there exist $A_{1}, A_{2} \in C(I,[0,+\infty))$ with $A_{1}(t) \geq A_{2}(t) \geq 0$ for $t \in I$, and the positive linear operators $\mathcal{L}_{1}-\mathcal{L}_{2}, \mathcal{L}_{1}+\mathcal{L}_{2} \in C(E, E)$ satisfy:

$$
\begin{aligned}
& Q_{i}(x, y)(t)-Q_{j}(v, w)(t) \\
\leq & A_{1}(t)[v(t)-x(t)]+A_{2}(t)[w(t)-y(t)]+\left[\mathcal{L}_{1}(v-x)\right](t)+\left[\mathcal{L}_{2}(w-y)\right](t)
\end{aligned}
$$

for $i \geq j, i, j=1,2$ for $t \in I, S \leq x \leq v \leq W, S \leq w \leq y \leq W ;\left(H_{3}\right)$ the functions $g_{1}, g_{2} \in C\left(\mathbb{R}^{4}, \mathbb{R}\right)$ are non-decreasing of the third variable and satisfy:

$$
g_{i}\left(\bar{x}_{1}, \bar{x}_{2}, y, y_{1}\right)-g_{j}\left(x_{1}, x_{2}, y, \bar{y}_{1}\right) \leq N_{1}\left(\bar{x}_{1}-x_{1}\right)-N_{2}\left(\bar{x}_{2}-x_{2}\right)+N_{3}\left(y_{1}-\bar{y}_{1}\right)
$$

for $i \geq j, i, j=1,2, S(0) \leq x_{1} \leq \bar{x}_{1} \leq W(0), S(0) \leq x_{2} \leq \bar{x}_{2} \leq W(0), S(Y) \leq y \leq \bar{y} \leq W(Y)$, $S(Y) \leq y_{1} \leq \bar{y}_{1} \leq W(Y)$, where $N_{1} \geq N_{2}+N_{3}, N_{2}>0, N_{3}>0$ and $\epsilon_{1}=\frac{N_{2}}{N_{1}}, \epsilon_{2}=\frac{N_{3}}{N_{1}}$.

Then, Problem (1) has solution $(x, y) \in[S, W] \times[S, W]$, where $[S, W]=\{\xi \in \Omega: S(t) \leq \xi(t) \leq$ $W(t), t \in I\}$.

Proof. Fist, using the following equations, one constructs two sequences $\left\{S_{n}(t)\right\}$ and $\left\{W_{n}(t)\right\}$ :

$$
\left\{\begin{array}{l}
S_{n}^{\prime}(t)=-B\left(S_{n}, W_{n}\right)(t)+F_{1}\left(S_{n-1}, W_{n-1}\right)(t), \quad t \in I \\
W_{n}^{\prime}(t)=-B\left(W_{n}, S_{n}\right)(t)+F_{2}\left(W_{n-1}, S_{n-1}\right)(t), \quad t \in I \\
N_{1} S_{n}(0)=N_{2} S_{n}(Y)-N_{3} W_{n}(Y)+\varphi_{1}\left(S_{n-1}, W_{n-1}\right) \\
N_{1} W_{n}(0)=N_{2} W_{n}(Y)-N_{3} S_{n}(Y)+\varphi_{2}\left(W_{n-1}, S_{n-1}\right)
\end{array}\right.
$$

with $n=1,2, \ldots$, where $S_{0}=S, W_{0}=W, F_{i}(x, y)(t)=Q_{i}(x, y)(t)+A_{1}(t) x(t)+A_{2}(t) y(t)+$ $\left(\mathcal{L}_{1} x\right)(t)+\left(\mathcal{L}_{1} y\right)(t)$ and $\varphi_{i}(u, v)=N_{1} u(0)-N_{2} u(Y)+N_{3} v(Y)-g_{i}(u(0), u(Y), v(0), v(Y)), i=1,2$

Apparently, from Theorem 1, Problem (12) has only one solution. Now, we are going to divide this proof into five parts.

Step 1. One shows that $S \leq S_{1}$ and $W_{1} \leq W$. 
Set $r(t)=S(t)-S_{1}(t), s(t)=W_{1}(t)-W(t)$. From $\left(H_{1}\right)$, we get:

$$
\begin{aligned}
r^{\prime}(t) & =S^{\prime}(t)-S_{1}^{\prime}(t) \\
& \leq Q_{1}(S, W)(t)+B\left(S_{1}, W_{1}\right)(t)-F_{1}(S, W)(t) \\
& \leq-A_{1}(t) r(t)+A_{2}(t) s(t)-\left(\mathcal{L}_{1} r\right)(t)+\left(\mathcal{L}_{2} s\right)(t), \quad t \in I, \\
s^{\prime}(t) & =W_{1}^{\prime}(t)-W^{\prime}(t) \\
& \leq B\left(W_{1}, S_{1}\right)(t)+F_{2}(W, S)(t)-Q_{2}(W, S)(t) \\
& \leq-A_{1}(t) s(t)+A_{2}(t) r(t)-\left(\mathcal{L}_{1} s\right)(t)+\left(\mathcal{L}_{2} r\right)(t), \quad t \in I, \\
r(0) & =S(0)-S_{1}(0) \\
& \leq \frac{1}{N_{1}} g 1(S(0), S(Y), W(0), W(Y))+\frac{N_{2}}{N_{1}} r(Y)+\frac{N_{3}}{N_{1}} s(Y) \\
& \leq \frac{N_{2}}{N_{1}} r(Y)+\frac{N_{3}}{N_{1}} s(Y),
\end{aligned}
$$

and:

$$
\begin{aligned}
s(0) & =W_{1}(0)-W(0) \\
& \leq-\frac{1}{N_{1}} g_{2}(W(0), W(Y), S(0), W(Y))+\frac{N_{2}}{N_{1}} s(Y)+\frac{N_{3}}{N_{1}} r(Y) \\
& \leq \frac{N_{2}}{N_{1}} s(Y)+\frac{N_{3}}{N_{1}} r(Y) .
\end{aligned}
$$

From Lemma 2 and $N_{1} \geq N_{2}>0, N_{1} \geq N_{3}>0$, we have $r(t) \leq 0$ and $s(t) \leq 0$, so $S \leq S_{1}$ and $W_{1} \leq W$.

Step 2. We show that $W_{n} \leq W_{n-1}$ and $S_{n-1} \leq S_{n}$ if $W_{n-1} \leq W_{n-2}, S_{n-2} \leq S_{n-1}, n=2,3, \ldots$

Set $e(t)=S_{n-1}(t)-S_{n}(t), l(t)=W_{n}(t)-W_{n-1}(t)$. From $\left(H_{1}\right),\left(H_{2}\right)$, we get:

$$
\begin{aligned}
e^{\prime}(t)= & S_{n-1}^{\prime}(t)-S_{n}^{\prime}(t) \\
= & -B\left(S_{n-1}, W_{n-1}\right)(t)+F_{1}\left(S_{n-2}, W_{n-2}\right)(t)+B\left(S_{n}, W_{n}\right)(t)-F_{1}\left(S_{n-1}, W_{n-1}\right)(t) \\
\leq & -A_{1}(t) e(t)+A_{2}(t) l(t)-\left(\mathcal{L}_{1} e\right)(t)+\left(\mathcal{L}_{2} l\right)(t)+Q_{1}\left(S_{n-2}, W_{n-2}\right) \\
& -Q_{1}\left(S_{n-1}, W_{n-1}\right)+A_{1}(t)\left[S_{n-2}(t)-S_{n-1}(t)\right]+A_{2}(t)\left[W_{n-2}(t)-W_{n-1}(t)\right] \\
& +\left[\mathcal{L}_{1}\left(S_{n-2}-S_{n-1}\right)\right](t)+\left[\mathcal{L}_{2}\left(W_{n-2}-W_{n-1}\right)\right](t) \\
\leq & -A_{1}(t) e(t)+A_{2}(t) l(t)-\left(\mathcal{L}_{1} e\right)(t)+\left(\mathcal{L}_{2} l\right)(t), \quad t \in I,
\end{aligned}
$$

and:

$$
\begin{aligned}
l^{\prime}(t)= & W_{n}^{\prime}(t)-W_{n-1}^{\prime}(t) \\
= & -B\left(W_{n}, S_{n}\right)(t)+F_{2}\left(W_{n-1}, S_{n-1}\right)(t)+B\left(W_{n-1}, S_{n-1}\right)(t)-F_{2}\left(W_{n-2}, S_{n-2}\right)(t) \\
\leq & -A_{1}(t) l(t)+A_{2}(t) e(t)-\left(\mathcal{L}_{1} l\right)(t)+\left(\mathcal{L}_{2} e\right)(t)+Q_{2}\left(W_{n-2}, S_{n-2}\right) \\
& -Q_{2}\left(W_{n-1}, S_{n-1}\right)+A_{1}(t)\left[W_{n-1}(t)-W_{n-2}(t)\right]+A_{2}(t)\left[S_{n-1}(t)-S_{n-2}(t)\right] \\
& +\left[\mathcal{L}_{1}\left(W_{n-1}-W_{n-2}\right)\right](t)+\left[\mathcal{L}_{2}\left(S_{n-1}-S_{n-2}\right)\right](t) \\
\leq & -A_{1}(t) l(t)+A_{2}(t) e(t)-\left(\mathcal{L}_{1} l\right)(t)+\left(\mathcal{L}_{2} e\right)(t), \quad t \in I .
\end{aligned}
$$


By $\left(H_{1}\right)$ and $\left(H_{3}\right)$, we have:

$$
\begin{aligned}
N_{1} e(0)= & N_{1}\left[S_{n-1}(0)-S_{n}(0)\right] \\
= & N_{1} S_{n-1}(0)-N_{2} S_{n}(Y)+N_{3} W_{n}(Y)-\varphi_{1}\left(S_{n-1}, W_{n-1}\right) \\
= & N_{2} e(Y)+N_{3} l(Y)+g_{1}\left(S_{n-1}(0), S_{n-1}(Y), W_{n-1}(0), W_{n-1}(Y)\right) \\
& -g_{1}\left(S_{n-2}(0), S_{n-2}(Y), W_{n-2}(0), W_{n-2}(Y)\right)+N_{1}\left[S_{n-2}(0)-S_{n-1}(0)\right] \\
& +N_{2}\left[S_{n-1}(Y)-S_{n-2}(Y)\right]+N_{3}\left[W_{n-2}(Y)-W_{n-1}(Y)\right] \\
\leq & N_{2} e(Y)+N_{3} l(Y),
\end{aligned}
$$

and:

$$
\begin{aligned}
N_{1} l(0)= & N_{1}\left[W_{n}(0)-W_{n-1}(0)\right] \\
= & N_{2} W_{n}(Y)-N_{3} S_{n}(Y)+\varphi_{2}\left(W_{n-1}, S_{n-1}\right)-N_{1} W_{n-1}(0) \\
= & N_{2} l(Y)+N_{3} e(Y)-g_{2}\left(W_{n-1}(0), W_{n-1}(Y), S_{n-1}(0), S_{n-1}(Y)\right) \\
& +g_{2}\left(W_{n-2}(0), W_{n-2}(Y), S_{n-2}(0), S_{n-2}(Y)\right)+N_{1}\left[W_{n-1}(0)-W_{n-2}(0)\right] \\
& -N_{2}\left[W_{n-1}(Y)-W_{n-2}(Y)\right]+N_{3}\left[S_{n-1}(Y)-S_{n-2}(Y)\right] \\
\leq & N_{2} l(Y)+N_{3} e(Y) .
\end{aligned}
$$

Using Lemma 2 and $N_{1} \geq N_{2}>0, N_{1} \geq N_{3}>0$, we have $e \leq 0, l \leq 0$, so $W_{n} \leq W_{n-1}, S_{n-1} \leq S_{n}$.

From mathematical induction, one gets that $\left\{S_{n}\right\}$ is a nondecreasing sequence, and $\left\{W_{n}\right\}$ is a nonincreasing sequence.

Step 3. We claim that $S_{1} \leq W_{1}$ if $S \leq W$.

Let $u(t)=S_{1}(t)-W_{1}(t)$. Using $\left(H_{2}\right)$ and $\left(H_{3}\right)$, one receives:

$$
\begin{aligned}
u^{\prime}(t)= & S_{1}^{\prime}(t)-W_{1}^{\prime}(t) \\
= & -B\left(S_{1}, W_{1}\right)(t)+F_{1}(S, W)(t)+B\left(W_{1}, S_{1}\right)(t)-F_{2}(W, S)(t) \\
= & -\left[A_{1}(t)-A_{2}(t)\right] u(t)-\left[\left(\mathcal{L}_{1}-\mathcal{L}_{2}\right) u\right](t)+Q_{1}(S, W)(t)+A_{1}(t) S(t) \\
& +A_{2}(t) W(t)+\left(\mathcal{L}_{1} S\right)(t)+\left(\mathcal{L}_{2} W\right)(t)-Q_{2}(W, S)(t) \\
& -A_{1}(t) W(t)-A_{2}(t) S(t)-\left(\mathcal{L}_{1} W\right)(t)-\left(\mathcal{L}_{2} S\right)(t) \\
\leq & -\left[A_{1}(t)-A_{2}(t)\right] u(t)-\left[\left(\mathcal{L}_{1}-\mathcal{L}_{2}\right) u\right](t), \quad t \in I,
\end{aligned}
$$

and:

$$
\begin{aligned}
N_{1} u(0)= & N_{1}\left[S_{1}(0)-W_{1}(0)\right] \\
= & \left(N_{2}+N_{3}\right) u(Y)+\varphi_{1}(S, W)-\varphi_{2}(W, S) \\
= & \left(N_{2}+N_{3}\right) u(Y)+N_{1}[S(0)-W(0)]+\left(N_{2}+N_{3}\right)[W(Y)-S(Y)] \\
& -g_{1}(S(0), S(Y), W(0), W(Y))+g_{2}(W(0), W(Y), S(0), S(Y)) \\
\leq & \left(N_{2}+N_{3}\right) u(Y) .
\end{aligned}
$$

From Lemma 1 , one arrives at $u \leq 0$, and $S_{1} \leq W_{1}$. Utilizing the mathematical induction, we gain $W_{n} \leq S_{n}, n=1,2, \ldots$

Step 4 . By the above three steps, we obtain:

$$
S_{0} \leq S_{1} \leq \cdots \leq S_{n} \leq \cdots \leq W_{n} \leq \cdots \leq W_{1} \leq W_{0},
$$


and every $S_{n}, W_{n}$ satisfies (12) for all $n$. We can get that the sequences $\left\{S_{n}(t)\right\},\left\{W_{n}(t)\right\}$ are monotonically and bounded. Then, there are $\rho, r$ satisfying that $\lim _{n \rightarrow \infty} S_{n}(t)=\rho(t), \lim _{n \rightarrow \infty} W_{n}(t)=r(t)$ and satisfying the equations:

$$
\left\{\begin{array}{l}
\rho^{\prime}(t)=Q_{1}(\rho, r)(t), \quad t \in I, \\
r^{\prime}(t)=Q_{2}(r, \rho)(t), \quad t \in I, \\
g(\rho(0), \rho(Y), r(0), r(Y))=0, \\
g(r(0), r(Y), \rho(0), \rho(Y))=0 .
\end{array}\right.
$$

It proves that a pair $(\rho, r)$ is the solution of Problem (1).

Step 5. One verifies that $(\rho, r)$ is an extremal solution of $(1)$ in $[S, W]$.

Let $(x, y)$ be any pair of solutions for (1) satisfying $S(t) \leq x(t), y(t) \leq W(t)$. We assume that there is a positive integer $n$ satisfying $S_{n-1}(t) \leq x(t), y(t) \leq W_{n-1}(t)$. Set $u(t)=S_{n}(t)-x(t)$ and $v(t)=y(t)-W_{n}(t)$. By using $\left(H_{2}\right)$ and $\left(H_{3}\right)$, one gets:

$$
\begin{aligned}
u^{\prime}(t)= & S_{n}^{\prime}(t)-x^{\prime}(t) \\
= & -B\left(S_{n}, W_{n}\right)(t)+F_{1}\left(S_{n-1}, W_{n-1}\right)-Q_{1}(x, y)(t) \\
= & -A_{1}(t) S_{n}(t)-A_{2}(t) W_{n}(t)-\left(\mathcal{L}_{1} S_{n}\right)(t)-\left(\mathcal{L}_{2} W_{n}\right)(t)+Q_{1}\left(S_{n-1}, W_{n-1}\right)(t) \\
& +A_{1}(t) S_{n-1}(t)+A_{2}(t) W_{n-1}(t)+\left(\mathcal{L}_{1} S_{n-1}\right)(t)+\left(\mathcal{L}_{2} W_{n-1}\right)(t)-Q_{1}(x, y)(t) \\
\leq & -A_{1}(t) u(t)+A_{2}(t) v(t)-\left(\mathcal{L}_{1} u\right)(t)+\left(\mathcal{L}_{2} v\right)(t), \quad t \in I, \\
v^{\prime}(t)= & y^{\prime}(t)-W_{n}^{\prime}(t) \\
= & Q_{2}(x, y)(t)+B\left(W_{n}, S_{n}\right)(t)-F_{2}\left(W_{n-1}, S_{n-1}\right) \\
= & Q_{2}(y, x)(t)+A_{1}(t) W_{n}(t)+A_{2}(t) S_{n}(t)+\left(\mathcal{L}_{1} W_{n}\right)(t)+\left(\mathcal{L}_{2} S_{n}\right)(t) \\
& -Q_{2}\left(W_{n-1}, S_{n-1}\right)(t)-A_{1}(t) W_{n-1}(t)-A_{2}(t) S_{n-1}(t) \\
& -\left(\mathcal{L}_{1} W_{n-1}\right)(t)-\left(\mathcal{L}_{2} S_{n-1}\right)(t) \\
\leq & -A_{1}(t) v(t)+A_{2}(t) u(t)-\left(\mathcal{L}_{1} v\right)(t)+\left(\mathcal{L}_{2} u\right)(t), \quad t \in I, \\
N_{1} u(0)= & N_{1}\left[S_{n}(0)-x(0)\right] \\
= & N_{2} S_{n}(Y)-N_{3} W_{n}(Y)+\varphi_{1}\left(S_{n-1}, W_{n-1}\right)-N_{1} x(0) \\
= & N_{2} S_{n}(Y)-N_{3} W_{n}(Y)+N_{1} S_{n-1}(0)-N_{2} S_{n-1}(Y)+N_{3} W_{n-1}(Y)-N_{1} x(0) \\
& -g_{1}\left(S_{n-1}(0), S_{n-1}(Y), W_{n-1}(0), W_{n-1}(Y)\right)+g_{1}(x(0), x(Y), y(0), y(Y)) \\
\leq & N_{2} S_{n}(Y)-N_{2} x(Y)-N_{3} W_{n}(Y)+N_{3} y(Y) \\
\leq & N_{2} u(Y)+N_{3} v(Y),
\end{aligned}
$$

and:

$$
\begin{aligned}
N_{1} v(0)= & N_{1}\left[y(0)-W_{n}(0)\right] \\
= & N_{1} y(0)-N_{2} W_{n}(Y)+N_{3} S_{n}(Y)+\varphi_{1}\left(W_{n-1}, S_{n-1}\right) \\
= & N_{1} y(0)-N_{2} W_{n}(Y)+N_{3} S_{n}(Y)-N_{1} W_{n-1}(0)+N_{2} W_{n-1}(Y)-N_{3} S_{n-1}(Y) \\
& +g_{1}\left(W_{n-1}(0), W_{n-1}(Y), S_{n-1}(0), S_{n-1}(Y)\right)-g_{1}(y(0), y(Y), x(0), x(Y)) \\
\leq & -N_{2} W_{n}(Y)+N_{2} y(Y)+N_{3} S_{n}(Y)-N_{3} x(Y) \\
\leq & N_{2} v(Y)+N_{3} u(Y) .
\end{aligned}
$$

From Lemma 2, one see that $S_{n}(t) \leq x(t), y(t) \leq W_{n}(t)$ on $I$. Since $S(t) \leq x(t), y(t) \leq W(t)$, by induction, one gets $S_{n}(t) \leq x(t), y(t) \leq W_{n}(t)$ for every $n \in \mathbb{N}$. Now, take the limit as $n \rightarrow \infty$, then $\rho(t) \leq x(t), y(t) \leq r(t)$. The proof is over. 


\section{Example}

Example 1. Assume that:

$$
\left\{\begin{array}{l}
z^{\prime}(t)=Q_{1}(z, w)(t), \quad t \in[0, Y] \\
w^{\prime}(t)=Q_{2}(w, z)(t), \quad t \in[0, Y] \\
g_{1}(z(0), z(Y), w(0), w(Y))=0 \\
g_{2}(w(0), w(Y), z(0), z(Y))=0
\end{array}\right.
$$

with:

$$
\begin{aligned}
& Q_{1}(z, w)(t)=-t z(t)-\frac{Y}{5 t} \int_{0}^{t} z(s) d s+\frac{3 Y}{10 t^{3}} \int_{0}^{t} s^{2} w(s) d s, \\
& Q_{2}(w, z)(t)=-t w(t)-\frac{Y}{5 t} \int_{0}^{t} w(s) d s+\frac{3 Y}{10 t^{3}} \int_{0}^{t} s^{2} z(s) d s, \\
& g(z(0), z(Y), w(0), w(Y))=4 z(0)-2 z(Y)+w(Y)-2, \\
& g(w(0), w(Y), z(0), z(Y))=4 w(0)-2 w(Y)+z(Y)-2 .
\end{aligned}
$$

Set $S=0, W=2$. It is easily verified that $W$ is an upper solution, $S$ is a lower solution and $S \leq W$. Take $\left(\mathcal{L}_{1} z\right)(t)=\frac{Y}{5 t} \int_{0}^{t} z(s) d s,\left(\mathcal{L}_{2} w\right)(t)=\frac{3 Y}{10 t^{3}} \int_{0}^{t} s^{2} w(s) d s, A_{1}(t)=t, A_{2}(t)=0, N_{1}=4, N_{2}=2$, $N_{3}=1$ and $Y=\frac{1}{2}$. It is easy to see that $\left(H_{1}\right),\left(H_{2}\right)$ and $\left(H_{3}\right)$ are satisfied. By simple computation, we obtain $\mathcal{L}_{1}(t)=0.2 Y, \mathcal{L}_{2}(t)=0.1 Y$, and:

$$
\begin{aligned}
& \int_{0}^{Y}\left[A_{1}(t)+A_{2}(t)+\mathcal{L}_{1}(t)+\mathcal{L}_{2}(t)\right] d t=0.2<\frac{N_{2}-N_{3}}{N_{1}}=0.25, \\
& \int_{0}^{Y}\left[A_{1}(t)-A_{2}(t)+\mathcal{L}_{1}(t)-\mathcal{L}_{2}(t)\right] d t=0.15<\frac{N_{2}+N_{3}}{N_{1}}=0.75, \\
& \frac{N_{1}}{N_{1}-\left(N_{2}-N_{3}\right) e^{-\int_{0}^{Y}\left[A_{1}(r)+A_{2}(r)\right] d r}}\left\|\mathcal{L}_{1}+\mathcal{L}_{2}\right\| Y<1, \\
& \frac{N_{1}}{N_{1}-\left(N_{2}+N_{3}\right) e^{-\int_{0}^{Y}\left[A_{1}(r)+A_{2}(r)\right] d r}}\left\|\mathcal{L}_{1}-\mathcal{L}_{2}\right\| Y<1 .
\end{aligned}
$$

Hence, utilizing Theorem 2, (13) has an extreme solution in $[S, W] \times[S, W]$.

This equation can be used as a simple model of the physical field, and this example shows that the conclusion in this paper applies to Problem (13).

\section{Conclusions}

In this paper, we obtain the existence results of coupled causal differential equations by means of the monotone iterative technique. In practice, after modeling in our field, if such coupled differential equations are obtained, we do not have to calculate the solution; we only need to use the sufficient conditions in this paper to judge the existence of the solution.

Author Contributions: W.-L.W. completed the proof and wrote the initial draft. J.-F.T. and W.-S.C. provided some problems and gave some suggestions of amendment. W.-L.W. then finalized the paper. All authors read and approved the final paper.

Funding: This work was supported by the Fundamental Research Funds for the Central Universities (No. 2015ZD29).

Acknowledgments: The authors are grateful to the reviewers for their suggestions, which improved the paper.

Conflicts of Interest: The authors declare no conflict of interest.

\section{References}

1. Drici, Z.; McRae, F.A.; Devi, J.V. Monotone iterative technique for periodic boundary value problems with causal operators. Nonlinear Anal. 2006, 64, 1271-1277. [CrossRef] 
2. Geng, F. Differential equations involving causal operators with nonlinear periodic boundary conditions. Math. Comput. Model. 2008, 48, 859-866. [CrossRef]

3. Lakshmikantham, V.; Leela, S.; Drici, Z.; McRae, F.A. Theory of Causal Differential Equations; World Scientific Press: Paris, France, 2009.

4. Tian, J.; Wang, W.; Cheung, W.S. Periodic boundary value problems for first-order impulsive difference equations with time delay. Adv. Differ. Equ. 2018, 2018, 79. [CrossRef]

5. Hu, Z.; Liu, W.; Chen, T. Existence of solutions for a coupled system of fractional differential equations at resonance. Bound. Value Probl. 2012, 2012, 98. [CrossRef]

6. Ntouyas, S.; Obaid, M. A coupled system of fractional differential equations with nonlocal integral boundary conditions. Adv. Differ. Equ. 2012, 2012, 130. [CrossRef]

7. Su, X. Boundary value problem for a coupled system of nonlinear fractional differential equations. Appl. Math. Lett. 2009, 22, 64-69. [CrossRef]

8. Jankowski, T. Existence of solutions for a coupled system of difference equations with causal operators. Appl. Math. Comput. 2013, 219, 9348-9355. [CrossRef]

(C) 2018 by the authors. Licensee MDPI, Basel, Switzerland. This article is an open access article distributed under the terms and conditions of the Creative Commons Attribution (CC BY) license (http://creativecommons.org/licenses/by/4.0/). 